ARTICLE

\title{
Structure of native glycolipoprotein filaments in honeybee royal jelly
}

Simone Mattei (1) ${ }^{1,2,4}$, Arvid Ban ${ }^{1,4}$, Armin Picenoni ${ }^{1}$, Marc Leibundgut ${ }^{1}$, Rudi Glockshuber (ib) ${ }^{1 凶} \&$ Daniel Boehringer (D) 1,3凶

Royal jelly (RJ) is produced by honeybees (Apis mellifera) as nutrition during larval development. The high viscosity of RJ originates from high concentrations of long lipoprotein filaments that include the glycosylated major royal jelly protein 1 (MRJP1), the small protein apisimin and insect lipids. Using cryo-electron microscopy we reveal the architecture and the composition of RJ filaments, in which the MRJP1 forms the outer shell of the assembly, surrounding stacked apisimin tetramers harbouring tightly packed lipids in the centre. The structural data rationalize the $\mathrm{pH}$-dependent disassembly of $\mathrm{RJ}$ filaments in the gut of the larvae.

\footnotetext{
${ }^{1}$ Department of Biology, Institute of Molecular Biology and Biophysics, ETH Zurich, Otto-Stern-Weg 5, Zurich 8093, Switzerland. ${ }^{2}$ Present address: Imaging Centre, European Molecular Biology Laboratory, Meyerhofstraße 1, 69117 Heidelberg, Germany. ${ }^{3}$ Present address: Cryo-EM Knowledge Hub, ETH Zurich, Otto-Stern-Weg 3, Zurich 8093, Switzerland. ${ }^{4}$ These authors contributed equally: Simone Mattei, Arvid Ban. ${ }^{\circledR}$ email: rudi@mol.biol.ethz.ch; boehringer@mol.biol.ethz.ch
} 
$\mathrm{R}$ oyal jelly (RJ) is a gelatinous, protein-, carbohydrate-, and lipid-rich secretion produced by the mandibular glands of nurse honeybees (Apis mellifera) ${ }^{1}$. In honeybee colonies, female castes are nutritionally regulated and only larvae exclusively fed on RJ develop into queens ${ }^{1-3}$. The major royal jelly glycoproteins in RJ are involved in cast determination of female larvae but the underlying mechanisms are still not fully understood $^{4-8}$. The major royal jelly protein 1 (MRJP1), a member of the family of yellow proteins with a molecular mass of $\sim 60 \mathrm{kDa}$, is the most abundant glycoprotein in $\mathrm{RJ}^{1,9}$. The $\mathrm{RJ}$ glycoproteins are secretory proteins of the hypopharyngeal glands ${ }^{10}$. At $\mathrm{pH}$ values below 4.5, as found in native RJ upon acidification in the mandibular glands ${ }^{11}$, MRJP1 assembles with the small $(5 \mathrm{kDa}) \mathrm{RJ}$ protein apisimin into long, hetero-polymeric filaments that can reach lengths above $1 \mu \mathrm{m}^{12}$. These filaments were recently shown to be responsible for the high viscosity of RJ, guaranteeing that RJ-embedded queen bee larvae remain stably attached to the ceiling of the vertically oriented queen bee cells ${ }^{12}$. The presently available structural information on MRJP1 is based on a crystal structure determined at neutral $\mathrm{pH}$, in which the MRJP1 was found in a planar, tetrameric complex together with four molecules of apisimin and eight molecules of 24methylenecholesterol ${ }^{13}$, consistent with the reported oligomeric state of MRJP1 in solution at neutral $\mathrm{pH}^{14}$. However, the architecture and composition of the MRJP1-containing filaments that form at the native acidic $\mathrm{pH} 4.0$ of $\mathrm{RJ}^{11}$ still remained unknown. Here we present the structure of native RJ filaments using a combination of cryogenic electron microscopy (cryo-EM) approaches based on tomography and helical reconstruction methods. The obtained reconstruction allowed us to define the composition and architecture of the native RJ filaments and to rationalize spontaneous $\mathrm{RJ}$ filament association at low $\mathrm{pH}$ and later dissociation at elevated $\mathrm{pH}$ values in the larvae gut.

\section{Results}

Purification of native RJ filaments. We purified RJ filaments from RJ by means of ammonium sulfate precipitation and size exclusion chromatography (SEC) at $\mathrm{pH} 4.0$ (see methods). SDSPAGE and mass spectrometry analysis confirmed that MRJP1 was the most abundant protein component of the filaments (Supplementary Fig. 1), which eluted at a SEC volume corresponding to a molecular mass above $500 \mathrm{kDa}$. In addition, Edman sequencing confirmed the presence of equimolar amounts of MRJP1 and apisimin in the RJ filament preparation. For our structural analysis we proceeded using the protein material from high-molecular mass SEC fractions containing the RJ filaments.

Cryo-electron tomography and subtomogram averaging reveal the architecture of RJ filaments. Purified RJ filaments were vitrified and imaged to acquire 11 dose-symmetric tilt series (Supplementary Fig. 2). From the reconstructed tomograms we could identify $241 \mathrm{RJ}$ filaments. An ab initio structure of the native filaments was determined by a reference-free, iterative alignment and averaging procedure of three-dimensional particles (subtomograms) that were extracted from one individual filament. The resulting low-resolution $a b$ initio reconstruction showed the overall architecture of the purified RJ filaments consisting of an $\mathrm{H}$-shaped unit oligomerizing into a symmetric helical assembly by stacking interactions (Supplementary Fig. 3). The available crystal structure of the D2 symmetric MRJP1 $1_{4} /$ apisimin $_{4} / 24$-methylenecholesterol ${ }_{8}$ oligomer could be fitted with confidence as rigid body within one $\mathrm{H}$-shaped unit. The RJ helical assembly retains the D2 point group symmetry of the tetramers since both of the 2 -fold rotational symmetry axes are orthogonal to the longitudinal axis of the filaments. Therefore, the RJ helical assembly has no polarity.

The alignment of the full dataset of 4045 subtomograms with applied D2 symmetry yielded a final reconstruction of the native RJ filament at $8 \AA$ resolution with clearly discernible secondary structure elements (Supplementary Fig. 3). By rigid-body fitting the crystallographic model of the crystallized RJ filament building block $^{13}$ into the calculated subtomogram averaging map we were able to reveal the architecture of the native RJ filaments that consist of symmetric $\mathrm{MRJP} 1_{4} /$ apisimin $_{4} / 24$-methylenecholesterol $_{8}$ units that are stacked to form a helical assembly with 54 A rise and $64^{\circ}$ rotation (Supplementary Fig. 3). Although the RJ helical assembly is overall linear and does not present supercoiling, the filaments observed in our tomograms show noticeable flexibility (Supplementary Fig. 2). This conformational landscape is also manifested as a considerable variation of the local resolution along the longitudinal axis of our subtomogram averaging map (Supplementary Fig. 3).

The high-resolution real-space helical reconstruction shows $N$ glycans and lipids within the RJ filament. To determine the structure at high resolution we used real-space helical reconstruction methods starting from accurate estimates of the helical rise and rotation angle determined from our subtomogram averaging reconstruction (Fig. 1a, d and Supplementary Fig. 4). The resulting map, calculated at $3.5 \AA$ resolution (Supplementary Figs. 4 and 5), was used for rebuilding the structure starting from the previous crystal structure and including the peripheral MRJP1 protein loops responsible for filament-forming inter-subunit contacts (Fig. 1 and Supplementary Figs. 6 and 7). Overall, we did not observe major conformational changes of MRPJ1 and apisimin in the RJ filaments with respect to the crystal structure determined at neutral $\mathrm{pH}$.

The MRJP1 primary structure contains three putative posttranslational $N$-glycosylation sites with the consensus sequences Asn-X-Ser or Asn-X-Thr. The glycosylation at these sites was suggested based on experiments that revealed a molecular mass shift from $57 \mathrm{kDa}$ to $47 \mathrm{kDa}$ after treatment of MRJP1 with $\mathrm{N}$ glycosidase $\mathrm{F}^{15}$, and based on the crystal structure of MRJP1-apisimin where electron density was observed for a $\mathrm{N}$ acetyl- $\beta$-D-glucosamine (NAG) residue at Asn144, even after deglycosylation by peptide: $N$-glycanase F. Our high-resolution map of the native MRJP1 filament shows clear densities branching from all three asparagine residues (Asn28, Asn144, and Asn177) predicted to have $N$-linked glycans (Supplementary Fig. 6). Although the intrinsic flexibility of the $N$-linked glycans limits the map resolution at those sites, we were able to model one NAG residue at Asn28, two NAG residues at Asn144, and two NAG residues plus a $\beta$-D-mannose (BMA) residue at Asn177 (Fig. 1c). Our structure thus provides structural evidence for these posttranslational modifications at all three predicted sites.

In addition to the N-linked glycans, we observed two unassigned, elongated densities at the core of the MRJP1 $1_{4} /$ apisimin $_{4} / 24$-methylenecholesterol ${ }_{8}$ oligomers (Supplementary Fig. $7 \mathrm{a}, \mathrm{b})$. The densities are $\sim 25 \AA$ long and are located at the center of the oligomer within a highly hydrophobic environment formed by the apisimin alpha helix 2 and the outer 24methylenecholesterol from each asymmetric unit of the tetrameric oligomer. Although building an atomic model for this region was not possible, the highly hydrophobic chemical environment and the shape and length of the observed densities are consistent with a fatty acid with aliphatic tails of 12-16 carbons, or possibly a mixture of those. Lipids are abundant in RJ, accounting for $3-6 \%$ of its wet weight ${ }^{16-19}$ and the release of these fatty acids in the midgut of the honeybee larvae following 


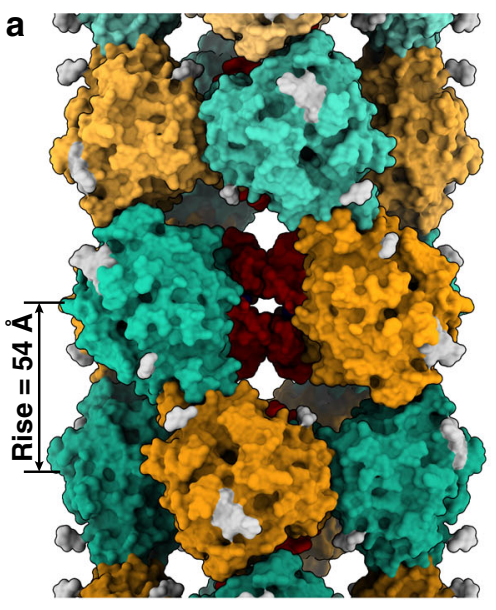

$\stackrel{90^{\circ}}{2}$

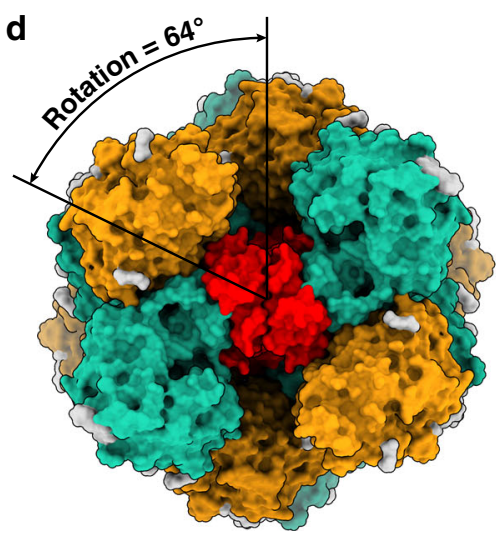

b

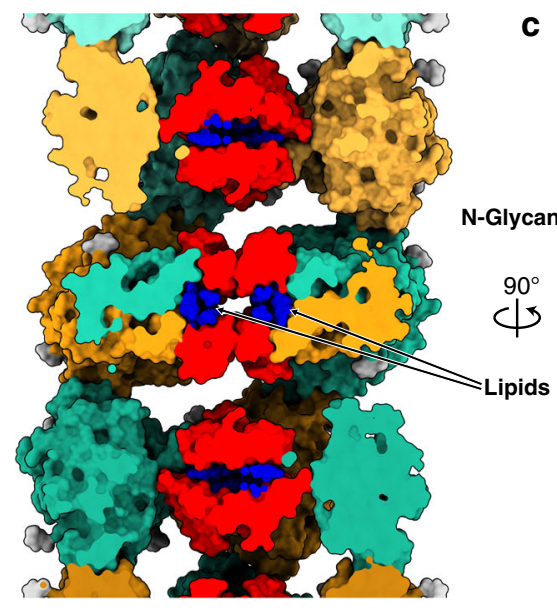

c

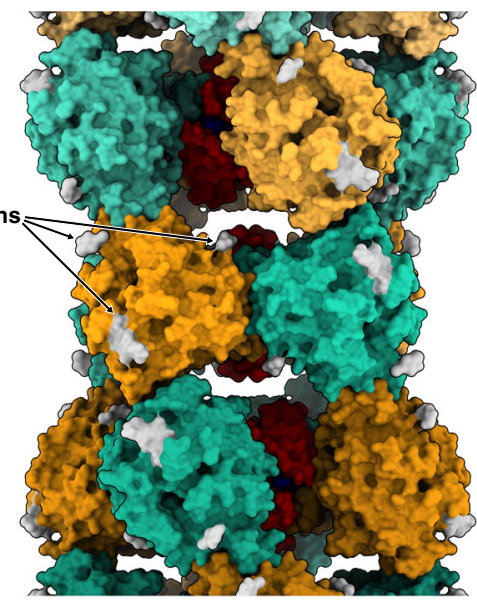

e

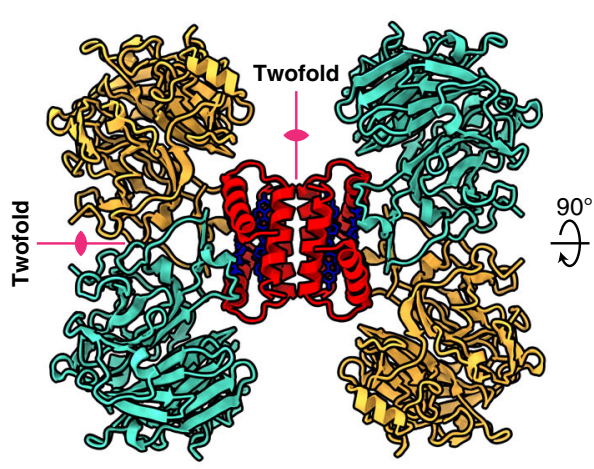

$\mathbf{f}$

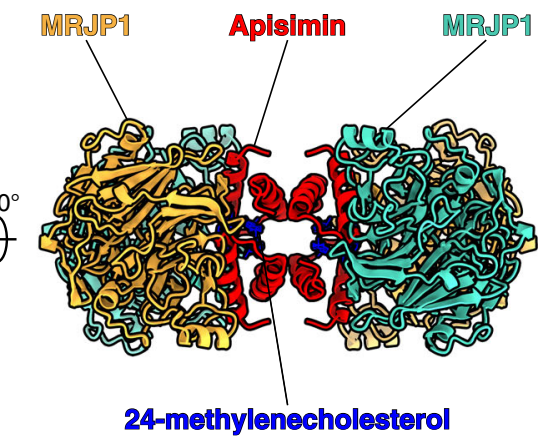

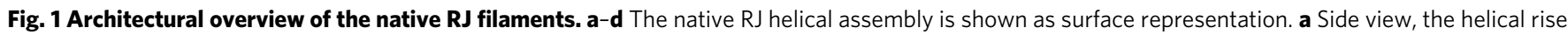
is shown. $\mathbf{b}$ Cross section along the longitudinal axis. c As in $\mathbf{a}$ after $90^{\circ}$ rotation along the longitudinal axis. $\mathbf{d}$ Top view, the helical rotation is shown. The MRJP1 subunits in each MRJP $_{4}$-apimisin 4 hetero-octamer layer are alternately colored in orange and cyan to distinguish subunits from different layers. Apisimin is colored in red, 24-methylenecholesterol in blue, and N-glycans in silver. e, $\mathbf{f}$ The H-shaped MRJP1 $4 /$ apisimin $4 / 24-$ methylenecholesterol $_{8}$ complex is shown from two orthogonal orientations with proteins represented as ribbons and the lipids as sticks. e Top view with the two 2 -fold symmetry axes indicated as pink lines. $\mathbf{f}$ Side view along one of the 2-fold axes.

the digestion of the RJ filaments has been suggested to play a role in the cast determination induced by the RJ diet ${ }^{20,21}$. The stoichiometric binding of lipids might play a role in the biogenesis of the $\mathrm{MRJP}_{4} /$ apisimin $_{4} / 24$-methylenecholesterol 8 filament units by stabilizing the interface between the apisimin subunits similar to the role of stoichiometrically bound lipids for assembly of $\mathrm{F}$ family pili $^{22}$.

Mechanism of RJ filament assembly at acidic pH. The stacked $\mathrm{MRJP}_{4} /$ apisimin $_{4} / 24$-methylenecholesterol ${ }_{8}$ building blocks are the basic RJ filament units that interact via two major interfaces involving a small number of electrostatic and hydrophobic interactions of MRJP1 (Fig. 2a, b). One of the contacts is mediated by homodimerization of MRJP1 alpha helices 1 ( $\alpha 1$, residues 47-56) from neighboring building blocks that mainly interact via hydrophobic interactions involving Gln51, Ile54, and Leu55 of helix 1 as well as Tyr61 in the following loop (Supplementary Fig. 7c). At the $N$-terminus of helix 1, Glu48 is located in close proximity to Phe395 and Asp396 of the neighboring RJ filament unit (Supplementary Fig. 7c). The helical assembly is further stabilized by a homodimerization interface present on the opposite side of the MRJP1 protein (Fig. 2a, b), involving homodimerization of a short turn (residues 280-281) followed by a loop segment (residues 287-295) that includes His294 and Asp292. This loop is partially disordered in the previously published crystal structure determined at neutral $\mathrm{pH}$, suggesting that it becomes ordered upon filament formation at acidic $\mathrm{pH}$.

Oligomerization of the RJ units into filaments is probably dependent on the protonation state of the glutamate residues $(\mathrm{pKa} \sim 4.3)^{23}$, which are mostly protonated at $\mathrm{pH} 4.0$. Upon exposure of MRJP1 to lower $\mathrm{pH}$ in the mandibular glands where $\mathrm{RJ}$ is produced, the electrostatic repulsion between the RJ filament units is lowered, allowing them to assemble into filaments via mostly hydrophobic contacts (Fig. 2b, c). RJ filament polymerization may proceed by addition of a new filament unit to either of the two ends of a growing filament. The loss of repulsive negative charges is the reason why close contacts that would be prohibited at neutral $\mathrm{pH}$ are observed between acidic residues, including Glu48 and Asp396. Additional stabilization of the filament at low $\mathrm{pH}$ likely arises from charge-charge interactions, including the interaction between His294 that is positively charged at low pH and Asp292 of MRJP1 on the neighboring $\mathrm{RJ}$ filament unit that would partially be negatively charged at $\mathrm{pH}$ 4.0. Although there is no sequence or structural similarity between any RJ proteins and spider silk proteins, the RJ filament formation is conceptually reminiscent to the self-assembly of spider silk proteins that occurs during secretion upon reduction 


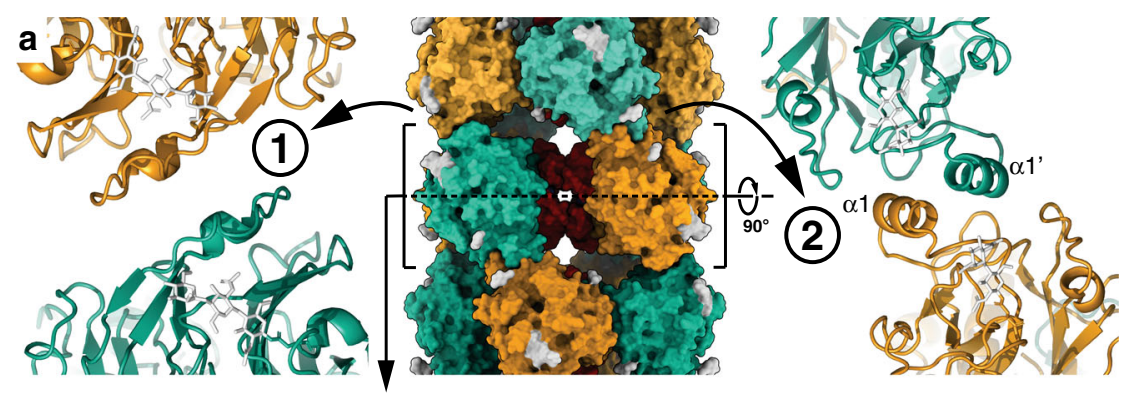

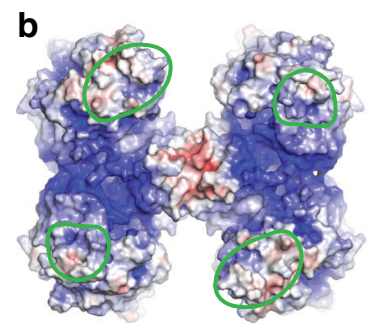

(1)

(2)

pH 4.0

C

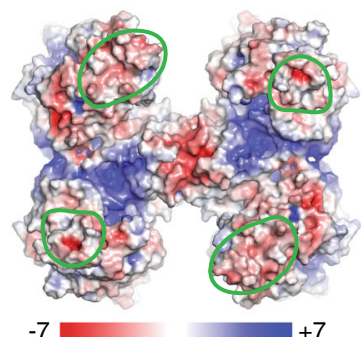

pH 5.5

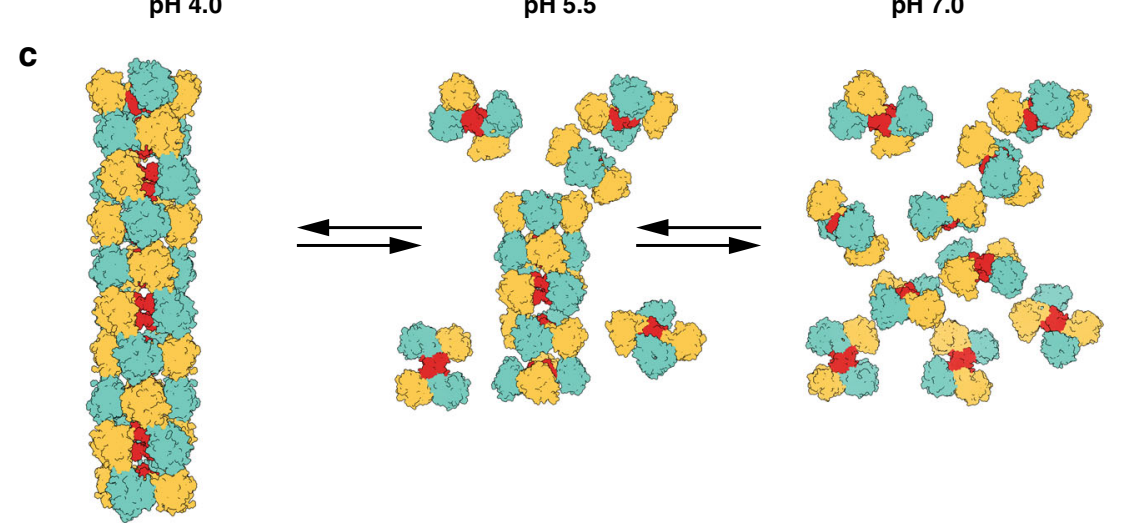

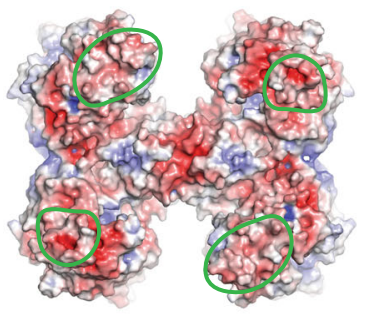

pH 7.0

Fig. 2 The pH-dependent disassembly of RJ filaments. a At pH 4.0, two major interfaces (labeled 1 and 2) between symmetry-related hetero-octamers exist in the RJ filament. Subunits (shown as surface in the overview and cartoons in the close-ups) are colored as in Fig. 1. The major contact (label 2) is mediated by homodimerization of MRJP1 alpha helices $1(\alpha 1)$. b Top views of the electrostatic surface potentials ( $\pm 7 \mathrm{kT} / \mathrm{e})$ of one hetero-octameric layer calculated at different $\mathrm{pH}$ values (see Methods for details). At pH 4.0, the four contact areas (1606 $\AA^{2}$ in total) between the hetero-octamer layers (encircled in green) are predominantly hydrophobic and allow stable assembly of the hetero-octamers into RJ filaments. At the higher $\mathrm{pH}$ values observed in the gut of bee larvae, the interaction sites become negatively charged. The repulsion between the hetero-octamer layers leads to destabilization and eventually disassembly of the RJ filament. c Schematics showing the $\mathrm{pH}$-dependent assembly model of RJ filaments.

of $\mathrm{pH}$ and involves protonation of glutamate residues ${ }^{24-26}$. Once $\mathrm{RJ}$ filaments are ingested by the bee larvae, the high $\mathrm{pH}$ found in the honeybee midgut will induce the disassembly of RJ filaments, destabilization of the MRJP1 protein ${ }^{27}$, and lead to its proteolytic degradation by digestive enzymes.

In conclusion, our structural analysis provides a comprehensive description of the architecture of the glycolipoprotein RJ filaments, and suggests a role of electrostatic interactions in their $\mathrm{pH}$-dependent assembly upon secretion from the mandibular glands of the honeybee and later disassembly in the midgut of the honeybee larvae.

\section{Methods}

RJ filaments purification. RJ filaments were purified from commercially available RJ (Bio-Gelée Royale (100\% pure, 15\% protein content), Naturwaren-Niederrhein $\mathrm{GmbH}, \mathrm{D}-47574$ Goch-Asperden, Germany). Briefly, a sample with an estimated $8 \mathrm{mg} / \mathrm{ml}$ total RJ protein concentration was prepared by dissolving $2.67 \mathrm{~g}$ of RJ in $50 \mathrm{ml}$ of water. The sample was dialyzed overnight at $4{ }^{\circ} \mathrm{C}$ using a $3.5 \mathrm{kDa}$ molecular weight cut-off membrane against 51 of $10 \mathrm{mM}$ formic acid-NaOH pH 4.0. The total protein was isolated by ammonium sulfate precipitation by adding $39 \mathrm{~g}$ of solid $\left(\mathrm{NH}_{4}\right)_{2} \mathrm{SO}_{4}$ to $64 \mathrm{ml}$ of dialyzed $\mathrm{RJ}$ solution. The sample was incubated at $4{ }^{\circ} \mathrm{C}$ for $2 \mathrm{~h}$ under light stirring. Precipitated RJ proteins were pelleted by centrifugation in a Sorvall SS34 rotor at $47,850 \times g$ for $60 \mathrm{~min}$. The supernatant was discarded and the protein pellet was dissolved in $21 \mathrm{ml}$ of $10 \mathrm{mM}$ formic acid- $\mathrm{NaOH} \mathrm{pH} 4.0$. The dissolved RJ protein sample was dialyzed against $5 \mathrm{l}$ of the same buffer for $4 \mathrm{~h}$ to remove the remaining ammonium sulfate and then filtered through a $0.2 \mu \mathrm{m}$ pore filter prior to gel filtration. $60 \mathrm{mg}$ of total protein were separated on a Superdex 200 $26 / 60$ gel filtration column ( $320 \mathrm{ml}$ volume) equilibrated with $10 \mathrm{mM}$ formic acid$\mathrm{NaOH} \mathrm{pH} \mathrm{4.0,150} \mathrm{mM} \mathrm{NaCl}$. Eluted proteins were detected via their absorbance at $280 \mathrm{~nm}$, and fractions of $5 \mathrm{ml}$ were collected. The fractions just outside of the exclusion volume $(\mathrm{C} 1-\mathrm{C} 4)$ were pooled for the structural analysis of the highmolecular mass RJ filaments (Supplementary Fig. 1a). According to molecular mass standards for calibration of the same gel filtration column, the pooled fractions corresponded to a molecular mass above $500 \mathrm{kDa}$.

Edman sequencing. The presence of apisimin and the molar MRJP1/apimisin ratio in purified RJ filaments was measured by quantitative Edman sequencing (Alphalyse A/S, Odense, Denmark) after calibration of HPLC peak intensities with an equimolar mixture of the 20 natural amino acids. Six consecutive degradation cycles confirmed the six $\mathrm{N}$-terminal residues of MRJP1 (NILRGE) and apisimin (KTSISV). The two residues identified in each degradation step always proved to be the most abundant degradation products. The average molar ratio between the respective MRJP1 and apisimin residue in these six degradation steps was $1.15 \pm$ 0.32 , confirming the 1:1 ratio between MRJP1 and apisimin in the RJ filament structure within experimental error.

SDS-PAGE and mass spectrometry (MS). The protein content of samples collected after each step of protein purification was analyzed by SDS-PAGE and MS. 
The protein samples were mixed with denaturing and reducing sample buffer and heated at $95{ }^{\circ} \mathrm{C}$ for $10 \mathrm{~min}$ prior to loading. The samples were then loaded on a polyacrylamide gel consisting of a stacking portion ( $4 \%$ acrylamide, $0.1 \%$ SDS, and $0.125 \mathrm{M}$ Tris-HCl buffer $\mathrm{pH}$ 6.8) layered on top of the resolving portion (15\% acrylamide, $0.1 \%$ SDS, and $0.4 \mathrm{M}$ Tris- $\mathrm{HCl}$ buffer $\mathrm{pH} 8.8$ ).

The gel was then stained with Coomassie blue to visualize the protein bands. In order to determine the identity of individual proteins in the fractions, protein bands were cut from the gel and sent for protein identification by MALDI-TOF mass spectrometry of tryptic peptides. Briefly, gel bands were cut into small pieces and washed twice with $100 \mu \mathrm{l}$ of $100 \mathrm{mM} \mathrm{NH}_{4} \mathrm{HCO}_{3} / 50 \%$ acetonitrile, then washed once with $50 \mu \mathrm{l}$ of acetonitrile. The supernatant was discarded and the bands were incubated at $60{ }^{\circ} \mathrm{C}$ for $34 \mathrm{~min}$ with $10 \mu \mathrm{l}$ of trypsin solution $(5 \mathrm{ng} / \mu \mathrm{l}$ in $10 \mathrm{mM}$ Tris$\mathrm{HCl} / 2 \mathrm{mM} \mathrm{CaCl} 2, \mathrm{pH} 8.2)$ and $30 \mu \mathrm{l}$ buffer $\left(10 \mathrm{mM}\right.$ Tris- $\mathrm{HCl} / 2 \mathrm{mM} \mathrm{CaCl}_{2}, \mathrm{pH}$ 8.2). After incubation, the supernatant was removed and the gel pieces extracted with $150 \mu \mathrm{l}$ of $0.1 \% \mathrm{TFA} / 50 \%$ acetonitrile for $15 \mathrm{~min}$ by ultrasonic treatment. Samples were dried and dissolved in $20 \mu \mathrm{l}$ of $0.1 \%$ trifluoroacetic acid (TFA). $1 \mu \mathrm{l}$ of each sample was mixed 1:1 with MALDI matrix solution $(1.4 \mathrm{mg} / \mathrm{ml} \mathrm{HCCA}$ in $85 \%$ acetonitrile, $0.1 \%$ TFA, $1 \mathrm{mM} \mathrm{NH} \mathrm{H}_{2} \mathrm{PO}_{4}$ ) and spotted on the target. Database searches were performed by using the Mascot (Swiss-Prot, TrEMBL) search programs. The main protein band was confirmed to be MRJP1, the other two prominent bands running above the MRJP1 band and below the MRJP1 band in the total protein lane labeled RJ (Supplementary Fig. 1b) were determined to be MRJP3 and MRJP2, respectively.

Cryo-EM sample preparation. A solution of $0.2 \mathrm{mg} / \mathrm{ml}$ graphene oxide was applied to glow discharged Quantifoil R2/2 grids and incubated for $90 \mathrm{~s}$. The excess of graphene oxide solution was removed by paper blotting and the grids were airdried. $5 \mu$ of sample solution from gel filtration fractions C1-C4 (Supplementary Fig. 1a) were applied to each graphene oxide-coated grid and plunge-frozen using a Vitrobot Mark IV (Thermo Fisher Scientific) operated at $12{ }^{\circ} \mathrm{C}$ with $95 \%$ humidity.

Cryo-EM data acquisition - tomography. Micrographs for electron cryotomography were acquired using a Titan Krios transmission electron microscope (Thermo Fisher Scientific) operated at $300 \mathrm{KeV}$, equipped with a Quantum postcolumn energy filter and a K2 direct electron detector (Gatan). Automated data acquisition was controlled using SerialEM ${ }^{28}$. A total of 11 tilt series were acquired at a nominal magnification of 105,000 x resulting in a calibrated pixel size of 1.387 $\AA ̊$. Tilt series were acquired using a dose-symmetric Hagen-scheme ${ }^{29}$ with a $3^{\circ}$ tilt increment and an angular range of $\pm 60^{\circ}$ and nominal defocus between -4.0 and $-6.3 \mu \mathrm{m}$. Each tilt image was exposed for $1.6 \mathrm{~s}$ for a total flux of $2.2 \mathrm{e}^{-} / \AA^{2}$, resulting in a total flux of $90 \mathrm{e}^{-} / \AA^{2}$ for a full tilt series of 41 micrographs. Each tilt image was acquired as a movie of four frames.

\section{Cryo-EM data processing - tomography}

Tomography. Movie frames of the tilt-series were aligned and averaged using of the MotionCor2 software ${ }^{30}$. Contrast transfer function (CTF) estimation of each motion-corrected tilt image was performed using CTFFIND4 ${ }^{31}$. Images were lowpass filtered according to the cumulative dose based on the previously published critical exposure curve ${ }^{32}$. Tilt series alignment and tomogram reconstruction were performed using $\mathrm{IMOD}^{33}$. Subsequent extraction, alignment, and averaging of three-dimensional particles (subtomograms) was performed using the Dynamo software package ${ }^{34}$. From the reconstructed tomograms, $241 \mathrm{RJ}$ filaments were identified and a total of 12,416 subtomograms were extracted along the spline of the filaments using a 3-fold oversampling with respect to the distance of the filament layers observable from the tomogram. An ab initio structure of the native RJ filaments was determined by a reference-free, iterative alignment and averaging procedure of 211 motion-corrected, dose-filtered, and CTF-corrected subtomograms that were extracted from one individual filament with a pixel size of $11.1 \AA$ and a box size of 48 pixels. The average of the extracted subtomograms was used as initial reference. The in-plane angular search range was $360^{\circ}$ with a $30^{\circ}$ step while the out-of-plane cone search range was $30^{\circ}$ with a $15^{\circ}$ step. Throughout the iterative alignment procedure, the subtomograms were aligned against a reference low-pass filtered at $40 \AA$, and all search angles were refined twice from the found local minima with final search steps of $7.5^{\circ}$ and $3.75^{\circ}$ for the in-plane search and the out-of-plane search, respectively. The obtained reference was then re-centered and re-oriented within its box to have the longitudinal axis of the filament aligned with the $z$-axis and intersecting the center of the box. Given the observed lack of filament polarity, the 12,416 subtomograms extracted from all filaments were then pooled together and iteratively aligned as described above. After alignment convergence, subtomograms that converged on overlapping positions (distance cutoff of 4 pixels corresponding to $44.4 \AA$ ) were removed. For each position only the subtomogram with the best cross-correlation score was retained. The remaining 4045 subtomograms were then re-extracted with a voxel size of $5.55 \AA$ using a box size of 64 pixels and iteratively aligned and averaged. The initial in-plane and outof-plane cone search ranges were $20^{\circ}$ with a $10^{\circ}$ step. Throughout the iterative alignment procedure, the subtomograms were aligned against a reference low-pass filtered at $40 \AA$ and all search angles were refined four times from the found local minima with final search step of $1.25^{\circ}$ for both the in-plane search and the out-ofplane search. The subtomograms were then extracted with a voxel size of $2.77 \AA$ using a box size of 96 pixels and split in two half-datasets that were further processed independently as "odd" and "even" datasets for gold standard procedure and aligned with applied D2 symmetry. The initial in-plane and out-of-plane cone search ranges were $10^{\circ}$ with a $5^{\circ}$ step. The subtomograms were aligned against a reference low-pass filtered at $17 \AA$ and all search angles were refined four times from the local minima with final search steps of $0.625^{\circ}$ for both the in-plane search and the out-of-plane search. The calculated FSC curve calculated by masking the central hetero-octameric subunit resulted in a final reconstruction of the native RJ filament at $8.8 \AA$ resolution using the $\mathrm{FSC}=0.143$ criterion.

Cryo-EM data acquisition - helical reconstruction. Micrographs for helical reconstruction were acquired using a Titan Krios transmission electron microscope (Thermo Fisher Scientific) operated at $300 \mathrm{KeV}$, equipped with a Quantum postcolumn energy filter and a K3 direct electron detector (Gatan). A total of 6656 micrographs were automatically collected using the SerialEM software ${ }^{28}$. For each micrograph a stack of 34 images was recorded in super-resolution mode at a nominal magnification of $105,000 \mathrm{x}$, corresponding to a calibrated pixel size of $0.42 \AA /$ pixel, with defocus ranging between -1 and $-3 \mu \mathrm{m}$. The total exposure time was $1.7 \mathrm{~s}$, resulting in a total electron dose of $\sim 82 \mathrm{e}^{-} / \AA^{2}$. Data acquisition parameters are summarized in Table S1.

Cryo-EM data processing - helical reconstruction. Movie frames acquired for helical reconstruction were motion-corrected, dose-weighted, and Fourier-cropped to a final pixel size of $0.84 \AA$ using MotionCor $2^{30}$. CTF parameters of non-doseweighted and motion-corrected micrographs were estimated using Gctf ${ }^{35}$. Motioncorrected micrographs were visually inspected to assess the quality of their power spectra and the distribution of RJ filaments, and 1990 micrographs were selected for further processing. Subsequent data processing was carried out using the implementation of the Iterative Helical Real Space Reconstruction (IHRSR) approach to helical processing within Relion $3.0^{36}$. Filaments were manually picked from 76 micrographs and 7004 particles were extracted with a box size of 512 pixels and rescaled by Fourier-cropping resulting in a final pixel size of $3.36 \AA$ and box size of 128 pixels. Initial 2D classification was performed to obtain class averages of the picked filaments. Four class averages were selected and used as templates to automatically pick 406,638 particles from the full dataset. The picked particles were extracted with a box size of 512 pixels applying a binning factor of 4 , resulting in a final pixel size of $3.36 \AA$ /pixel and a box size of 128 pixels. One round of $2 \mathrm{D}$ classification was used to remove featureless particles, and 270,434 particles were selected for further processing. 3D classification of the selected particles was performed applying the helical parameters obtained from the tomographic reconstruction and providing a featureless cylindrical density with a height of $430 \AA$ and an outer diameter of $370 \AA$ as reference. One class showing structural features comparable to the helical assembly obtained by subtomogram averaging was selected for further processing. The 240,483 particles assigned to the selected class were re-extracted with a box size of 512 pixels and rescaled by Fourier-cropping resulting in a final pixel size of $1.19 \AA$ and a box size of 360 pixels. The extracted particles were aligned using 3D refinement and submitted to a skip-alignment 3D classification focused on 8 MRJP1 subunits for further removal of misaligned particles. The refinement and post-processing of the 43,130 selected particles yielded a map resolved at $3.9 \AA$ resolution. After per-particle CTF refinement the structure was refined and resolved at $3.5 \AA$ resolution. (See Supplementary Fig. 4 for the processing scheme and Supplementary Fig. 5 for the quality of the cryoEM map).

Model building and refinement. For atomic interpretation of the $3.5 \AA$ cryo-EM map (Supplementary Fig. 5), one MRJP ${ }_{1} /$ apisimin $_{1}$ protomer of the published crystal structure (PDB 5yyl) ${ }^{13}$ was docked into the central, best-resolved area of the map using UCSF ChimeraX ${ }^{37}$ and adjusted in COOT $^{38}$. Unassigned areas of density, including non-modeled side chains, missing protein loops and residues at the $N$-terminus of MRJP1, a sulfate ion, and glycosylation sites were built manually. The final model of the protomer included residues 20-431 of MRJP1 and residues 33-76 of apisimin. After symmetry expansion to a model comprising four heterooctameric $\mathrm{MRJP}_{4} /$ apisimin $_{4}$ layers, the coordinates were subjected to five cycles of real space refinement using PHENIX ${ }^{39}$, during which protein secondary structure, side chain rotamer, and Ramachandran restraints as well as strict NCS between protomers were applied (Table S1). For PDB deposition, the two central heterooctameric layers were kept and re-refined for an additional cycle to update the B factors and model statistics, while the two peripheral layers located in less wellordered areas of EM density were removed. The geometry of the final model was validated using MolProbity. The real space correlation coefficient $\left(\mathrm{CC}_{\text {mask }}\right)$ between the cryo-EM map and the refined model was 0.83 , indicating an excellent fit, and the model-versus-map FSC at the FSC $=0.5$ criterion resulted in a similar resolution as the one calculated from the map half-sets at the $\mathrm{FSC}=0.143$ criterion (Supplementary Fig. 5E).

Surface potential calculation. The surface potentials shown in Fig. 2 were calculated with the PDB2PQR server ${ }^{40,41}$ using the AMBER forcefield ${ }^{42}$ in conjunction with PROPKA ${ }^{43-45}$ to assign the protonation state at the provided pHs. The surface potentials were visualized in PyMOL (The PyMOL Molecular Graphics 
System, Version 2.0 Schrödinger, LCC) using the $\mathrm{APBS}^{41}$ plugin with a color gradient from $-7 \mathrm{kT} / \mathrm{e}$ (red) to $+7 \mathrm{kT} / \mathrm{e}$ (blue). The calculations were performed using one entire hetero-octameric layer, while cofactors and glycosyl moieties were excluded. For histidines built in alternative conformations, only one of the rotamers was included at full occupancy.

Reporting summary. Further information on research design is available in the Nature Research Reporting Summary linked to this article.

\section{Data availability}

Cryo-EM maps and atomic models have been deposited in the Electron Microscopy Data Bank (EMDB) and wwPDB, respectively, with the following accession code EMD-11892 and PDB 7ASD (RJ filament real space helical reconstruction); EMD-11898 (tomographic reconstruction of RJ filaments). Other data are available from the corresponding authors upon reasonable request.

Received: 23 September 2020; Accepted: 13 November 2020; Published online: 08 December 2020

\section{References}

1. Buttstedt, A., Moritz, R. F. A. \& Erler, S. Origin and function of the major royal jelly proteins of the honeybee (Apis mellifera) as members of the yellow gene family. Biol. Rev. Camb. Philos. Soc. 89, 255-269 (2013).

2. Rembold, H. \& Dietz, A. In Vitamins and Hormones, Vol. 23 (eds Harris, R. S., Wool, I. G. \& Loraine, J. A.) (Academic Press, 1966).

3. Haydak, M. H. Honey Bee Nutrition. Annu. Rev. Entomol. 15, 143-156 (1970).

4. Kamakura, M. Kamakura replies. Nature 537, E13-E13 (2016).

5. Kamakura, M. Royalactin induces queen differentiation in honeybees. Nature 473, 478-483 (2011)

6. Buttstedt, A., Ihling, C. H., Pietzsch, M. \& Moritz, R. F. A. Royalactin is not a royal making of a queen. Nature 537, E10-E12 (2016).

7. Kucharski, R., Foret, S. \& Maleszka, R. EGFR gene methylation is not involved in Royalactin controlled phenotypic polymorphism in honey bees. Sci. Rep. 5, 14070 (2015).

8. Maleszka, R. Beyond Royalactin and a master inducer explanation of phenotypic plasticity in honey bees. Commun. Biol. 1, 8 (2018).

9. Hanes, J. \& Šimuth, J. Identification and partial characterization of the major royal jelly protein of the honey bee (Apis mellifera L.). J. Apicultural Res. 31, 22-26 (1992)

10. Knecht, D. \& Kaatz, H. Patterns of larval food production by hypopharyngeal glands in adult worker honey bees. Apidologie 21, 457-468 (1990).

11. Hoffman, I. Untersuchungen uber die Herkunft der Komponenten des Koniginnenfuttersaftes der Honigbienen. Naturwiss 47, 239-240 (1960)

12. Buttstedt, A. et al. How honeybees defy gravity with royal jelly to raise queens. Curr. Biol. 28, 1095-1100 (2018).

13. Tian, W. et al. Architecture of the native major royal jelly protein 1 oligomer. Nat. Commun. 9, 3373 (2018).

14. Mandacaru, S. C. et al. Characterizing the structure and oligomerization of major royal jelly protein 1 (MRJP1) by mass spectrometry and complementary biophysical tools. Biochemistry 56, 1645-1655 (2017)

15. Ohashi, K., Natori, S. \& Kubo, T. Change in the mode of gene expression of the hypopharyngeal gland cells with an age-dependent role change of the worker honeybee Apis mellifera L. Eur. J. Biochem. 249, 797-802 (1997).

16. von Planta, A. Ueber den futtersaft der bienen. Biol. Chem. 12, 327-354 (1888).

17. Lercker, G., Capella, P., Conte, L. S., Ruini, F. \& Giordani, G. Components of royal jelly II. The lipid fraction, hydrocarbons and sterols. J. Apicultural Res. 21, 178-184 (1982).

18. Takenaka, T. \& Echigo, T. Proteins and peptides in royal jelly. Nippon Nōgeikagaku Kaishi 57, 1203-1209 (1983).

19. Howe, S. R., Dimick, P. S. \& Benton, A. W. Composition of freshly harvested and commercial royal jelly. J. Apicultural Res. 24, 52-61 (1985).

20. Spannhoff, A. et al. Histone deacetylase inhibitor activity in royal jelly might facilitate caste switching in bees. EMBO Rep. 12, 238-243 (2011).

21. Chan, Q. W. T. et al. Honey bee protein atlas at organ-level resolution. Genome Res. 23, 1951-1960 (2013).

22. Costa, T. R. D. et al. Structure of the bacterial sex F pilus reveals an assembly of a stoichiometric protein-phospholipid complex. Cell 166, 1436-1444 (2016).

23. Grimsley, G. R., Scholtz, J. M. \& Pace, C. N. A summary of the measured $\mathrm{pK}$ values of the ionizable groups in folded proteins. Protein Sci. 18, 247-251 (2009).

24. Askarieh, G. et al. Self-assembly of spider silk proteins is controlled by a pHsensitive relay. Nature 465, 236-238 (2010).
25. Kronqvist, N. et al. Sequential pH-driven dimerization and stabilization of the $\mathrm{N}$-terminal domain enables rapid spider silk formation. Nat. Commun. 5, 3254 (2014)

26. Hagn, F. et al. A conserved spider silk domain acts as a molecular switch that controls fibre assembly. Nature 465, 239-242 (2010).

27. Zheng, H., Powell, J. E., Steele, M. I., Dietrich, C. \& Moran, N. A. Honeybee gut microbiota promotes host weight gain via bacterial metabolism and hormonal signaling. Proc. Natl. Acad. Sci. 114, 4775-4780 (2017).

28. Mastronarde, D. N. Automated electron microscope tomography using robust prediction of specimen movements. J. Struct. Biol. 152, 36-51 (2005).

29. Hagen, W. J. H., Wan, W. \& Briggs, J. A. G. Implementation of a cryo-electron tomography tilt-scheme optimized for high resolution subtomogram averaging. J. Struct. Biol. 197, 191-198 (2017).

30. Zheng, S. Q. et al. MotionCor2: anisotropic correction of beam-induced motion for improved cryo-electron microscopy. Nat. Methods 14, 331-332 (2017).

31. Rohou, A. \& Grigorieff, N. CTFFIND4: fast and accurate defocus estimation from electron micrographs. J. Struct. Biol. 192, 216-221 (2015).

32. Grant, T. \& Grigorieff, N. Measuring the optimal exposure for single particle cryo-EM using a $2.6 \AA ̊$ reconstruction of rotavirus VP6. eLife 4, e06980 (2015)

33. Kremer, J. R., Mastronarde, D. N. \& McIntosh, J. R. Computer visualization of three-dimensional image data using IMOD. J. Struct. Biol. 116, 71-76 (1996).

34. Castano-Diez, D., Kudryashev, M., Arheit, M. \& Stahlberg, H. Dynamo: a flexible, user-friendly development tool for subtomogram averaging of cryoEM data in high-performance computing environments. J. Struct. Biol. 178, 139-151 (2012).

35. Zhang, K. Gctf: real-time CTF determination and correction. J. Struct. Biol. 193, 1-12 (2016)

36. Zivanov, J. et al. New tools for automated high-resolution cryo-EM structure determination in RELION-3. eLife 7, e42166 (2018).

37. Goddard, T. D. et al. UCSF ChimeraX: meeting modern challenges in visualization and analysis. Protein Sci. 27, 14-25 (2018)

38. Emsley, P., Lohkamp, B., Scott, W. G. \& Cowtan, K. Features and development of Coot. Acta Crystallogr. Sect. D. 66, 486-501 (2010).

39. Afonine, P. V. et al. New tools for the analysis and validation of cryo-EM maps and atomic models. Acta Crystallogr. Sect. D. 74, 814-840 (2018).

40. Dolinsky, T. J. et al. PDB2PQR: expanding and upgrading automated preparation of biomolecular structures for molecular simulations. Nucleic Acids Res. 35, W522-W525 (2007).

41. Dolinsky, T. J., Nielsen, J. E., McCammon, J. A. \& Baker, N. A. PDB2PQR: an automated pipeline for the setup of Poisson-Boltzmann electrostatics calculations. Nucleic Acids Res. 32, W665-W667 (2004).

42. Ponder, J. W. \& Case, D. A. Advances in Protein Chemistry, Vol. 66 (Academic Press, 2003).

43. Rostkowski, M., Olsson, M. H. M., Søndergaard, C. R. \& Jensen, J. H. Graphical analysis of $\mathrm{pH}$-dependent properties of proteins predicted using PROPKA. BMC Struct. Biol. 11, 6-6 (2011).

44. Søndergaard, C. R., Olsson, M. H. M., Rostkowski, M. \& Jensen, J. H. Improved treatment of ligands and coupling effects in empirical calculation and rationalization of pKa values. J. Chem. Theory Comput. 7, 2284-2295 (2011).

45. Olsson, M. H. M., Søndergaard, C. R., Rostkowski, M. \& Jensen, J. H. PROPKA3: consistent treatment of internal and surface residues in empirical pKa predictions. J. Chem. Theory Comput. 7, 525-537 (2011).

\section{Acknowledgements}

We thank Rafal Zdanowicz and Fabia Canonica for help with the biochemical work. We thank Serge Chesnov (Functional Genomics Center Zurich) for mass spectrometry analysis. We thank Kun Qu for providing the plugins used for lattice maps visualization in UCSF Chimera. We thank Dr. Anja Nickel Lutz from Literargymnasium Raemibuehl Zurich, for scientific discussions and suggestions. The cryo-EM data were collected at the Scientific Center for Optical and Electron Microscopy (ScopeM) at ETH Zurich, and we thank M. Peterek for his support. The cryo-EM data were processed on the Leonhard computing cluster of the Swiss National Supercomputing Centre (CSCS). S.M. was supported by a European Molecular Biology Organization (EMBO) long-term fellowship (ALTF 793-2017) and a Human Frontier Science Program long-term fellowship (LT000008/2018-L)

\section{Author contributions}

R.G. initiated the project and R.G. and D.B. coordinated the project. A.B. and R.G. purified the sample. D.B., A.B., and A.P. prepared samples for cryo-EM. S.M., D.B., and A.P. acquired cryo-EM data. S.M., D.B., and A.B. obtained cryo-EM reconstructions. S.M., M.L., and A.B. built and refined an atomic model. All authors contributed to the final version of the manuscript.

\section{Competing interests}

The authors declare no competing interests. 


\section{Additional information}

Supplementary information is available for this paper at https://doi.org/10.1038/s41467020-20135-x

Correspondence and requests for materials should be addressed to R.G. or D.B.

Peer review information Nature Communications thanks Katrina Brudzynski and the other, anonymous, reviewer(s) for their contribution to the peer review of this work.

Reprints and permission information is available at http://www.nature.com/reprints

Publisher's note Springer Nature remains neutral with regard to jurisdictional claims in published maps and institutional affiliations. (c) (i) Open Access This article is licensed under a Creative Commons Attribution 4.0 International License, which permits use, sharing, adaptation, distribution and reproduction in any medium or format, as long as you give appropriate credit to the original author(s) and the source, provide a link to the Creative Commons license, and indicate if changes were made. The images or other third party material in this article are included in the article's Creative Commons license, unles indicated otherwise in a credit line to the material. If material is not included in the article's Creative Commons license and your intended use is not permitted by statutory regulation or exceeds the permitted use, you will need to obtain permission directly from the copyright holder. To view a copy of this license, visit http://creativecommons.org/ licenses/by/4.0/.

(C) The Author(s) 2020 